\title{
Objective Trade-off in MPC Based Energy Management for Microgrids
}

\author{
Dominik Mildt, Marco Cupelli, and Antonello Monti \\ Institute for Automation of Complex Power Systems \\ E.ON Energy Research Center RWTH Aachen University, Aachen, Germany \\ [dmildt, mcupelli, amonti]@eonerc.rwth-aachen.de
}

\begin{abstract}
Microgrids (MGs) are considered one of the key enabling factors of future electric power systems. Their limited size allows optimal control, as the solution space of optimization problems increases exponentially with the number of assets, if significant model simplifications are not undertaken. Additionally, the capability to disconnect from the main grid and autonomously supply loads can serve to increase the resilience to failures in the main grid and be provided as a service to the Distribution System Operator (DSO). An energy management system (EMS) is responsible to facilitate the optimal use of resources; however, optimality can refer to a multitude of target objectives. This paper builds up on previous research, implementing a Model Predictive Control (MPC) based EMS that is used to show the trade-off between four different objectives in MG operation. The different objective functions are the minimization of operational cost, minimization of the energy exchanged with the main grid, minimization of resistive losses and maximization of potential islanding time (PIT) for a possible future islanding event.
\end{abstract}

Index Terms-- Energy Management, Islanding, Microgrid, Model Predictive Control, Multi-Objective Optimization

\section{INTRODUCTION}

Microgrids (MGs) represent parts of the electric power system that aggregates distributed energy resources (DERs) and loads and connects them to the remaining grid only through a switch at a point of common coupling (PCC) [1]. MGs have been identified as one of the key enabling factors of active distribution systems [1]. The aggregation of connected assets allows optimal coordination for a feasible number of entities [2]. Furthermore the capability to disconnect from the main grid increases resilience to technical problems as well as the reliability of load supply for consumers [1].

MG control assumes a hierarchical structure [3] in which the highest level is given by a tertiary controller, that is responsible for operation of the switch to the main grid. It usually encompasses an energy management system (EMS) that is used to determine optimal operation and coordination of all DERs. Optimality always refers to a set operational objective, of which numerous can be defined for MGs. The authors in [3] classify objectives into economic, environmental and technical aspects. The contrast between local and system level objectives in general smart distribution systems is explained in [4]. Weighting of several objectives in a multi-objective optimization has become a state-of-the-art for EMS [5]. A general introduction to multi-objective optimization techniques is found in [6]. Among recent works, [7] analyzes the tradeoff in priority-based scheduling, while [8] compares different scenarios in a linear weighted optimization.
This work extends on the findings in [9], where the maximization of the Potential Islanding Time (PIT) was added for the first time to the pool of EMS objectives, i.e. maximizing the time that the MG could disconnect from the main grid, if an islanding command was received. The EMS presented in [9]-[10] is extended, specifically to include a much more accurate approximation of the cost of battery energy storage system (BESS) wear [11], as compared to the simplified linear and quadratic functions of power which are employed by the majority of works. Parameter variation is performed for a weighted linear combination of four objectives for a $48 \mathrm{~h}$ simulation of a generic MG test system. Analysis shows the trade-off between specific objectives.

Chapter II briefly introduces the developed EMS. Chapter III explains the compared objective functions. Chapter IV outlines the simulation setup. Chapter V provides simulation results and chapter IV finally gives a short conclusion.

\section{EMS DEFINITION}

The proposed EMS employs a Model Predictive Control (MPC) scheme regarding a control horizon of discrete time steps $t \in\{1, \ldots, T\}$ of equal length $\Delta \mathrm{t}$. It is developed for a generic low voltage (LV) MG consisting of busses $i \in \mathcal{N}$ and lines $(i, j) \in \mathcal{E}$ between busses $i$ and $j$, where bus $i=$ 1 is the PCC. A radial feeder structure is assumed, which is typically the case in LV distribution grids. This allows the integration of an exact relaxation of the power flow constraints in their DistFlow formulation [12]. The overall problem is then posed as a Mixed Integer Second Order Cone Problem (MISOCP) [9][10]. The radial grid topology can be mapped to a connected, tree-structured graph where the nodes equal to the busses and edges equal to the lines. For each line $(i, j) \in \mathcal{E}$ in the given grid, let $l_{i, j}(t)$ denote the squared current magnitude. The complex line impedance is given as $z_{i, j}=r_{i, j}+j x_{i, j}$, separated into resistance and reactance. Analogously, $S_{i, j}(t)=P_{i, j}(t)+$ $j Q_{i, j}(t)$ is the complex power flow from bus $i$ to $j$, given as active and reactive power values. Similarly, for each system bus $i \in \mathcal{N}, v_{i}(t)$ denotes its squared voltage magnitude and $s_{i}(t)=p_{i}(t)+j q_{i}(t)$ its complex net power injection. The convex relaxation of the power flow for node $j$ with parent node $i$ is then stated by:

$$
\begin{gathered}
p_{j}(t)=\left(r_{i, j} l_{i, j}(t)-P_{i, j}(t)\right)+\sum_{k:(j, k) \in \mathcal{E}} P_{j, k}(t), \\
q_{j}(t)=\left(x_{i, j} l_{i, j}(t)-Q_{i, j}(t)\right)+\sum_{k:(j, k) \in \mathcal{E}} Q_{j, k}(t),
\end{gathered}
$$




$$
\begin{gathered}
v_{j}(t)=v_{i}(t)-2\left(r_{i, j} P_{i, j}(t)+x_{i, j} Q_{i, j}(t)\right) \\
+\left(r_{i, j}^{2}+x_{i, j}^{2}\right) l_{i, j}(t), \\
l_{i, j}(t) \geq \frac{P_{i, j}(t)^{2}+Q_{i, j}(t)^{2}}{v_{i}(t)} .
\end{gathered}
$$

The constraint relaxation is expressed in (4) where the inequality sign replaces equality. Power $p_{1}(t)$ is the power exchanged with the main grid, i.e. the difference between imported power $P_{\text {imp }}(t)$ and exported power $P_{\text {exp }}(t)$. Simultaneous import and export is avoided by introducing the binary parameter $\delta_{\text {exp }}(t)$ :

$$
\begin{gathered}
p_{1}(t)=P_{\text {imp }}(t)-P_{\text {exp }}(t), \\
0 \leq P_{\text {exp }}(t) \leq \delta_{\text {exp }}(t) P_{\text {Grid }}^{\text {Max }}, \\
0 \leq P_{\text {exp }}(t) \leq\left(1-\delta_{\text {exp }}(t)\right) P_{\text {Grid }}^{\text {Max }} .
\end{gathered}
$$

When disconnected from the main grid, the $\mathrm{MG}$ is assumed to operate with a single device at bus $r$ acting as a grid forming element, providing a new voltage reference. This setup is often preferred by the distribution system operators (DSOs) and MG operators as it enables a simpler configuration of the grid and avoid the necessity of droop control between all DERs [9]. The binary parameter $\delta_{P C C}(t)$ is introduced to indicate whether the $\mathrm{MG}$ is connected to the remaining system at time $t$. To accommodate islanded operation, eq. (3) is modified for the relationship between busses 1 and 2 , turning it into conditional constraint (9). This introduces also the alternative voltage reference (10). Conditional statements in optimization problem can be modelled through a combination of binary parameters and big-M relaxations, which are left out for the sake of readability and can automatically formulated by appropriate environments [13]. Additionally, the power flowing through the grid connection must be equal to zero in islanded operation, which is handled implicitly by (8).

$$
\begin{aligned}
& -\delta_{P C C}(t) l_{1,2}^{M a x} \leq l_{1,2}(t) \leq \delta_{P C C}(t) l_{1,2}^{M a x}, \\
& \text { if } \delta_{P C C}(t)=1 \\
& v_{2}(t)=v_{1}(t)-2\left(r_{1,2} P_{1,2}(t)+x_{1,2} Q_{1,2}(t)\right) \\
& +\left(r_{1,2}^{2}+x_{1,2}^{2}\right) l_{1,2}(t) \\
& \text { if } \delta_{P C C}(t)=0 \\
& v_{r}(t)=(1 \text { p. u. })^{2} \text {. }
\end{aligned}
$$

More details on the modelling of assets and their implementation as constraints to the optimization problems are presented in [9] and [10]. Specifically, reactive power outputs are either defined by assuming that loads operate at a constant power factors or they are implicitly limited via maximum apparent power $S^{\text {Max }}$ of flexible assets and generation units:

$$
P^{2}+Q^{2} \leq S^{\operatorname{Max}}
$$

\section{OBJECTIVE FUNCTION}

As stated previously, operational objectives are compared by a parameter variation of scaling factors $\rho$ in a weighted linear combination. In total, four separate objectives are combined:

$$
J=\rho_{C} J_{C}+\rho_{P} J_{P}+\rho_{P I T} J_{P I T}+\rho_{\text {LosS }} J_{\text {LosS }} .
$$

Here:

- $\quad J_{C}$ and $\rho_{C}$ refer to the function of economic cost and its weight.

- $J_{P}$ and $\rho_{P}$ refer to the function of cumulative energy exchange with the main grid and is weight.

- $J_{P I T}$ and $\rho_{P I T}$ refer to the function introduced to maximize the PIT and its weight.

- $J_{\text {Loss }}$ and $\rho_{\text {Loss }}$ refer to the function of cumulative line losses and its weight.

\section{A. Economic Cost}

The economic cost of the MG over the horizon regarded by the MPC controller can be expressed as:

$$
\begin{aligned}
J_{C} & =\sum_{t=1}^{T}\left(c_{i m p}(t) P_{i m p}(t)-c_{\text {exp }}(t) P_{\text {exp }}(t)\right) \Delta t \\
& +\sum_{i \in \mathcal{N}} \sum_{t=1}^{T}\left(f_{B E S S, i}(t)+f_{M T, i}(t)+f_{F C, i}(t)\right),
\end{aligned}
$$

where $c_{\text {imp }}(t)$ and $c_{\text {exp }}(t)$ are the cost of imported and exported power at the time $t$. $f_{b a t, i}(t), f_{m t, i}(t)$ and $f_{f c, i}(t)$ define the cost functions of a battery energy storage systems (BESS), a microturbine (MT) and a fuel cell (FC) potentially connected to bus $i$ at time $t$. In the following, the subscript $i$ is omitted in cost functions of the individual assets to simplify notation.

Most recent publications on EMS model the cost of BESS behavior simply by linear or quadratic functions of power. In contrast, this paper employs the more refined approach introduced in [11], explicitly modeling discharging behavior in separate variables. The cost function is stated by the combination of three quadratic terms, penalizing deviation from a reference State Of Charge (SOC), BESS wear, related to charging or discharging power $P_{B E S S, c}(t)$ and $P_{B E S S, d}(t)$, and BESS wear related to the cyclic Depth Of Discharge (DOD). The cost coefficients $c_{S O C}, c_{B E S S, P}$ and $c_{D O D}$ can be extracted from technical data sheets.

$$
\begin{gathered}
f_{B E S S}(t)=c_{S O C}\left(S O C_{B E S S}(t)-S O C_{B E S S}^{r e f}\right)^{2} \\
+c_{B E S S, P}\left[\left(P_{B E S S, c}(t)\right)^{2}\right. \\
\left.+\left(P_{B E S S, d}(t)\right)^{2}\right] \\
+c_{D O D}\left[\left(D O D_{B E S S, c}(t)\right)^{2}\right. \\
\left.+\left(D O D_{B E S S, d}(t)\right)^{2}\right]
\end{gathered}
$$


The SOC, by slight misuse of notation, refers to the energy stored in the BESS relative to the maximum value $W_{B E S S}^{M a x}$ and propagates in time considering charging and discharging efficiencies $\eta_{B E S S, c}$ and $\eta_{B E S S, d}$ :

$$
\begin{aligned}
& S O C_{B E S S}(t+1)=S O C_{B E S S}(t) \\
& \quad+\frac{P_{B E S S, c}(t) \eta_{B E S S, c} \Delta t}{W_{B E S S}^{M a x}}-\frac{P_{B E S S, d}(t) \Delta t}{\eta_{B E S S, d} W_{B E S S}^{M a x}} .
\end{aligned}
$$

Charging and discharging power of the BESS are limited by respective maximum values $P_{B E S S, c}^{\text {Max }}$ and $P_{B E S S, d}^{M a x}$ and binary parameters $\delta_{B E S S, c}(t)$ that prevents concurrence:

$$
\begin{gathered}
0 \leq P_{B E S S, c}(t) \leq \delta_{B E S S, c}(t) P_{B E S S, c}^{M a x}, \\
0 \leq P_{B E S S, d}(t) \leq\left(1-\delta_{B E S S, c}(t)\right) P_{B E S S, d}^{M a x} .
\end{gathered}
$$

Cyclic DOD is split into two variables $D O D_{B E S S, C}$ and $D O D_{B E S S, d}$ for charging and discharging operation, coupled in their time propagation via $\delta_{B E S S, c}(t)$. In contrast to [11], an explicit idling state is omitted for the fact that the solver can change between operational modes employing a negligible power command, which can easily be resolved to idling behavior in a post processing step:

$$
\begin{aligned}
& \text { if } \delta_{B E S S, c}(t+1)=1 \\
& D O D_{B E S S, c}(t+1)=D O D_{B E S S, c}(t) \\
& +\frac{P_{B E S S, c}(t) \eta_{B E S S, c} \Delta t}{W_{B E S S}^{M a x}}, \\
& D O D_{B E S S, d}(t+1)=0 \text {. } \\
& \text { if } \delta_{B E S S, c}(t+1)=0 \\
& D_{B E S S, c}(t+1)=0 \text {, } \\
& D O D_{B E S S, d}(t+1)=D O D_{B E S S, d}(t) \\
& +\frac{P_{B E S S, d}(t) \Delta t}{\eta_{B E S S, d} W_{B E S S}^{M a x}} .
\end{aligned}
$$

The MT cost is modelled as a linear function of power output $P_{M T}(t)$ and a base cost for operation. Associated cost coefficients $c_{M T, l i n}$ and $c_{M T, \text { base }}$ by first calculating generation cost in relation to power output from fuel price and MT efficiency at different operating points. Then, a linear regression is performed. The binary parameter $\delta_{M T}(t)$ indicates whether the MT is operating at the time $t$ :

$$
\begin{aligned}
f_{M T}(t)= & \left(c_{M T, \text { base }} \delta_{M T}(t)+c_{M T, l i n} P_{M T}(t)\right) \Delta t, \\
& 0 \leq P_{M T}(t) \leq \delta_{M T}(t) P_{M T}^{M a x} .
\end{aligned}
$$

Constant efficiency is assumed for the FC which implies that the costs scale linearly with its power generation $P_{F C}(t)$.

$$
f_{F C}(t)=c_{F C} P_{F C}(t) \Delta t
$$

\section{B. Cumulative Energy Exchange}

The cumulative energy exchange with the main grid over horizon $T$ is characterized by:

$$
J_{P}=\sum_{t=1}^{T} p_{1}(t) \Delta t=\sum_{t=1}^{T}\left(P_{\text {imp }}(t)-P_{\text {exp }}(t)\right) \Delta t
$$

This objective corresponds to the maximization of the aggregated usage of local DER.

\section{PIT Maximization}

$J_{P I T}$ is introduced to maximize the resources available to allow a potential future islanding event. It minimizes the time steps the MG is connected to the main grid assuming an islanding event at a predefined, future time step $t_{\text {island }}$. This translates to optimizing reserves to facilitate a maximum PIT for this hypothetical islanding event.

$$
J_{P I T}=\sum_{t=t_{\text {island }}}^{T} \delta_{P C C}(t) .
$$

This work plans for an islanding event a single time step in the future which means that $t_{\text {island }}=2$. Immediate islanding could however also be modelled via the limiting case $t_{\text {island }}=1$. Additionally, consecutive islanding is ensured with the inequality below:

$$
\delta_{P C C}(t+1) \geq \delta_{P C C}(t) \quad \forall t \in\left[t_{\text {island }}, T\right] .
$$

\section{Loss Minimization}

Minimization of joule energy losses is one of the most frequently employed objectives in optimal power flow calculation and is here presented in the respective summation over all lines and all time steps $t$ :

$$
J_{\text {Loss }}=\sum_{(i, j) \in \mathcal{E}} \sum_{t=1}^{T} r_{i, j} l_{i, j}(t) \Delta t .
$$

\section{E. Objective normalization}

To reasonably combine objectives in the optimization, all individual objective terms must lie within the same order of magnitude. Therefore, normalizing constants are introduced on each objective, mapping them to the interval between 0 and 1 , before the weighting with the respective scaling factor $\rho$ :

$$
\begin{aligned}
J_{c, \text { norm }} & =\frac{J_{c}}{k_{c}}, & J_{P, \text { norm }} & =\frac{J_{P}}{k_{P}}, \\
J_{P I T, \text { norm }} & =\frac{J_{P I T}}{k_{P I T}}, & J_{\text {Loss }, \text { norm }} & =\frac{J_{\text {Loss }}}{k_{\text {Loss }}} .
\end{aligned}
$$

The normalizing constant $k_{c}$ for the cost function is calculated as the price incurred if the MG bought energy equivalent to the annual cumulative average demand of all loads $\bar{P} \approx 50 \mathrm{~kW}$ at each time step, not considering local generation and priced at annual average price $\bar{c}_{\text {imp }}=0.237$ $€$.

$$
k_{c}=T \bar{c}_{i m p} \bar{P} \Delta t
$$

The normalizing constant $k_{P}$ for the energy exchange minimization is defined as the energy imported if the MG were to import energy $\bar{P} \cdot \Delta t$ at each time step:

$$
k_{P}=T \bar{P} \Delta t \text {. }
$$

The normalizing constant $k_{P I T}$ for PIT maximization is defined as the maximum number of time steps, which could be scheduled for islanding:

$$
k_{P I T}=\left(T-t_{\text {island }}+1\right) .
$$




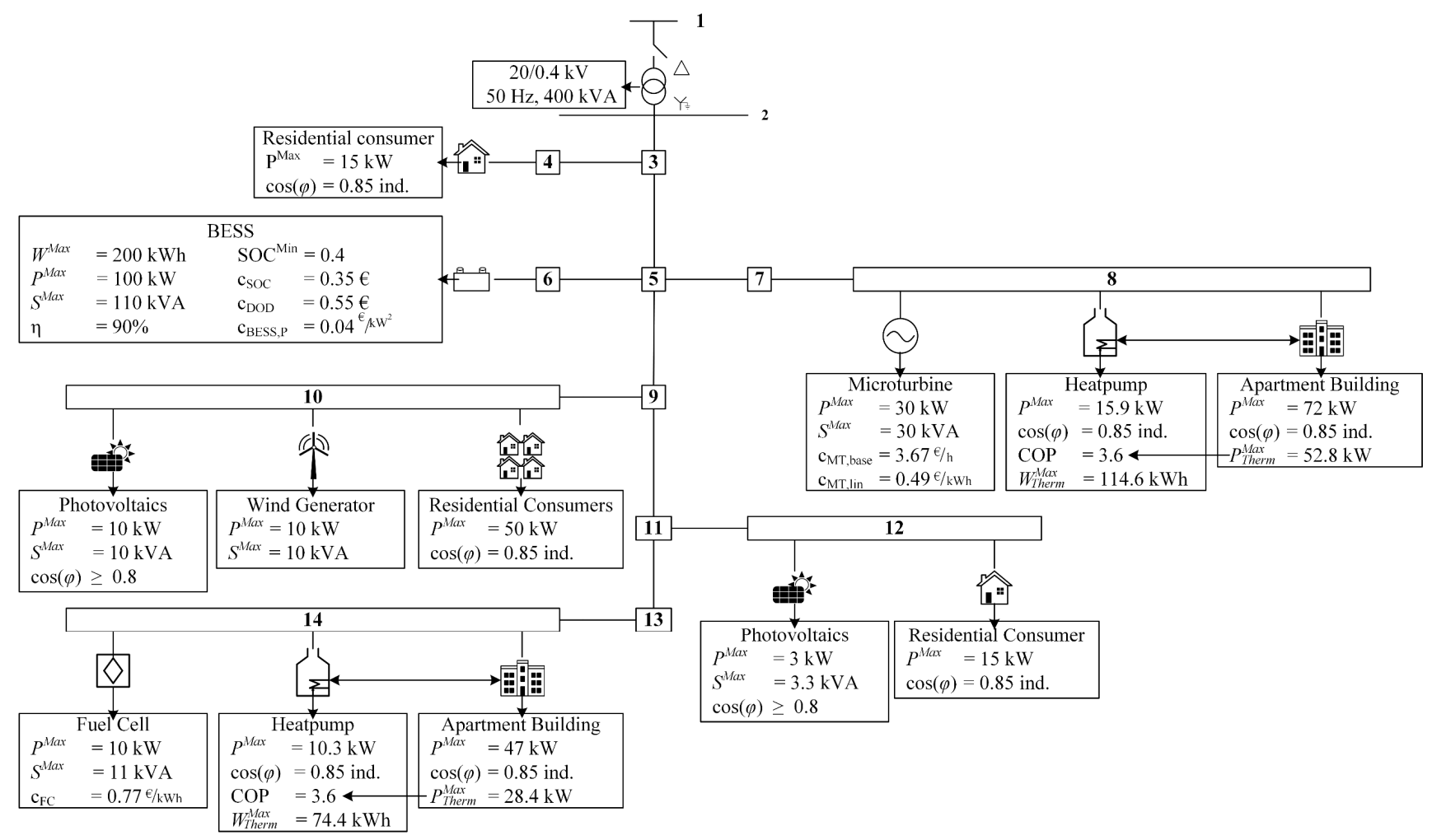

Fig. 1: MG reference setup

The normalizing constant $k_{\text {Loss }}$ is designed to be $4 \%$ of the constant $k_{P}$, which roughly fits the results obtained in previous simulations of the reference system:

$$
k_{\text {Loss }}=0.04 k_{P} \text {. }
$$

\section{SiMULATION SETUP}

The simulation was set up in MATLAB [14], making use of the Simscape Power Systems toolbox. Appliances were represented as constant power sources, switching their set points at each time step and propagated in time according to the simplified models used in the MPC formulation. The optimization problems were formulated in the YALMIP toolbox [13] and solved with commercial GUROBI software [15].

\section{F. MG Test System}

The regarded MG test system is based on the CIGRE LV MG benchmark [16] and was modified so that a larger variety of DER flexibility could be provided. In particular, the BESS size was increased to provide more resources for a potential islanding event and heat pumps were added to apartment buildings, representing electro-thermal coupling. A depiction of the grid is given in Fig. 1, also providing an overview of technical parameters and constraints. The superscript Max indicates maximum values for active power $P$, apparent power $S$ and stored energy $W$. When minimum values are not explicitly indicated with superscript Min, they were assumed as zero. The subscript Therm indicates that parameters refer to the thermal domain.
All input time series refer to a location in central Europe for the base year 2010 and were provided in hourly resolution. Residential consumer and apartment building load profiles were synthesized using occupancy-based statistic tools [17] and rescaled to meet the maximum annual demand $P^{\operatorname{Max}}$ as stated in Fig. 1. Thermal load profiles were created from 2010 German standard load profiles. Historic data of temperature, irradiation, wind speed and humidity were used to create generation profiles for photovoltaic and wind generation. Hourly electricity spot prices $c(t)$ were taken from [18] and then rescaled to meet the 2010 average consumer electricity price $\bar{c}=$ $0.2369 € / \mathrm{kWh}$. Import and export prices were then adjusted with scaling factors to $c_{\text {imp }}(t)=1.1 c(t)$ and to $c_{\text {exp }}(t)=0.9 c(t)$, for reducing simple arbitrage. To isolate the effect of the different objective combinations a perfect forecast was employed for all resources.

\section{G. Simulation Parameters}

The employed EMS was implemented with a forecast horizon of $T=24 \mathrm{~h}$ of equidistant time steps of length $\Delta t=1 \mathrm{~h}$. Operation was simulated for a period of $48 \mathrm{~h}$, representing the 2 . and 3. May 2010. These days were found to present a significant variety of operational patterns of all renewable energy resources and loads in previous, annual simulation of MG operation.

The objective weights $\rho_{C}, \rho_{P}, \rho_{P I T}$ and $\rho_{\text {Loss }}$ were each varied within the set $[1,2,6,24]$. This resulted in a total of $4^{4}=256$ combinations. However, some of these would deliver identical results, if the ratio between all weighting factors remains the same. Results were used to estimate 


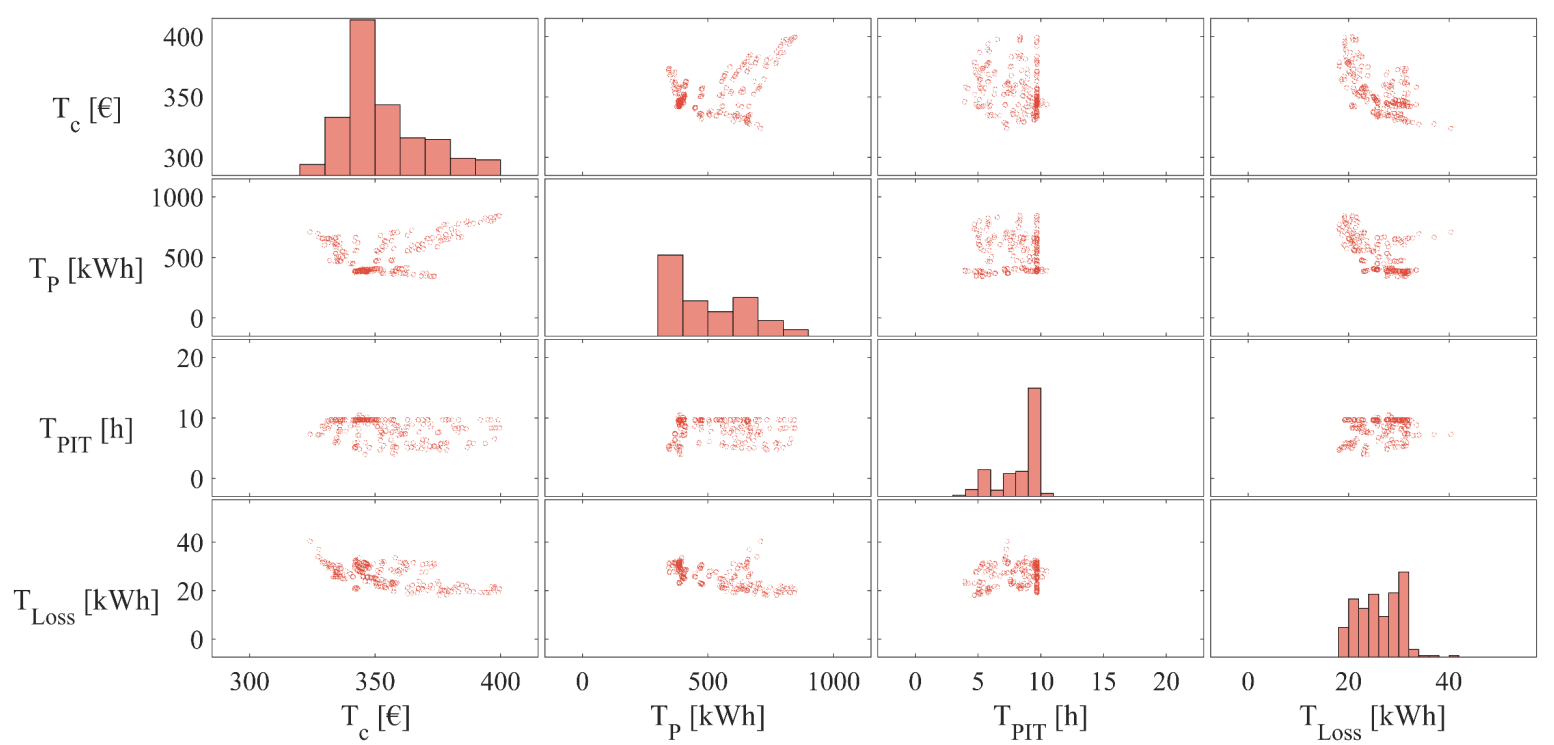

Fig 2.: Performance metrics of operational objectives plotted against each other

points on the pareto front [6] of the multi-objective optimization problem.

\section{RESULTS}

Four performance metrics are introduced to compare results of the simulations:

- $\quad T_{C}$ is the total cost incurred within the 48 -h period.

- $\quad T_{P}$ is the total net energy exchanged with the main grid within the 48-h period.

- $\quad T_{P I T}$ is the average PIT achievable at one timestep in the future, as planned by the EMS.

- $\quad T_{\text {Loss }}$ is the energy loss incurred within the 48-h period.

The performance metrics are chosen to evaluate results over the full simulation horizon in relation to individual objectives in a single number. They capture the underlying design principle of the associated objective functions and illustrate the trade-off to be made.

In Fig. 2 the performance metrics of all scenarios are plotted against each other in pairs. Each plot can be interpreted as the set of resulting pareto optimal points projected to a $2 \mathrm{D}$ coordinate system of two objectives.

Note that the plots on the main diagonal represent a histogram of the resulting values of the individual metrices

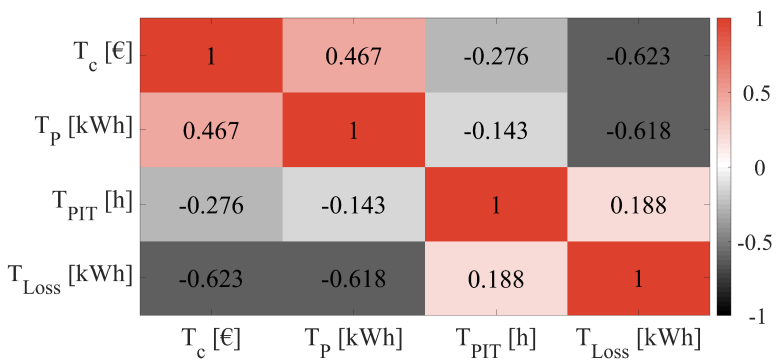

Fig 3.: Heatmap of correlation coefficients between performance metrices instead. Further, Fig. 3 provides a heatmap of the correlation coefficients between the performance metrics of all scenarios collected in vectors. They are used to visualize the relationship between objectives, leveraging statistical means.

First, Fig. 2 clearly shows that $T_{P I T}$ assumes a very similar value in more than half of all scenarios. This underlines the results presented in [9], that showed that PIT is often limited by peak demand hours of loads within the network, if installed load exceeds generation capacity in the MG.

Both cumulative cost $T_{C}$ and energy exchange $T_{P}$ appear to be negatively correlated with the average PIT $T_{P I T}$. This also corresponds well to the results obtained in [9]. It was shown that maximizing the PIT leads to a more conservative operation of energy storage to withhold resources to supply load. Additionally, the EMS fundamentally ignored the objective to withhold resources at time $t$, if it deemed disconnection from the main grid impossible at the next timestep $t_{\text {island }}=t+1$. Accordingly, energy storages would be rapidly depleted until islanding became possible again, causing excessive charging events at that time, i.e. high overall demand peaks. These events usually fell into the peak demand hours of the day, where electricity prices were high.

However, careful observation of Fig. 2 shows, that there are several cases, where the average PIT passes the threshold value of about $9.68 \mathrm{~h}$, incurring comparatively low cost and energy exchange. These turned out to be cases where only $\rho_{\text {PIT }}$ assumed the maximum value of 24 . On the one hand, this indicates, that if the MG is scheduled to withhold resources for islanding operation even in peak demand hours and plan for islanding operation, excessive load peaks can be avoided. These results are further underlined in Table 1. It shows mean PIT $\bar{T}_{P I T}$, mean SOC $\overline{S O C}_{B E S S, 6}$ of the BESS at bus 6 and mean peak power exchange with the main grid $\bar{p}_{1}^{\text {Max }}$, averaged respectively for all scenarios employing a set value of $\rho_{\text {PIT }}$.

On the other hand, it also hints towards difficulties in multi-objective optimization in the MPC formulation, 
when $J_{P I T}$ is the single discrete valued function. If islanding is deemed completely impossible by the EMS for only one single time step, it negatively influences $T_{C}$ and $T_{P}$ in the long run, even if both cost and energy exchange are lower for the specific optimization at that time step.

Another observation from Fig. 2, supported by the significant correlation coefficients found in Fig. 3, is that both cumulative cost $T_{C}$ and net energy exchange $T_{P}$ are negatively correlated with the cumulative losses $T_{\text {Loss }}$. These results may first appear counterintuitive. Energy losses equate additional active power demand that must be supplied by either DER or the main grid, i.e. also incurring cost. The reason is however found in the grid topology. The largest generator in the $\mathrm{MG}$ is connected to bus 8, which again connects to the central feeder as a side branch, compare Fig. 1. This implicates weaker wiring and a relatively higher resistance to bus 8 , compared to the main feeder running all the way to bus 14 . Along with increased focus on loss minimization, the generation of the MG at bus 8 is more often curtailed even if power could be exported to supply the remaining $\mathrm{MG}$, to reduce line currents. In reverse, more load is supplied from the main grid, which also proves more expensive at the given tariff. In a general sense, this indicates that loss minimization can in fact induce additional cost in a $\mathrm{MG}$, if wiring is heterogeneous and flexible DERs are connected to lines of relatively higher resistance.

Finally, Fig. 2 and 3 indicate that $T_{C}$ and $T_{P}$ mostly show a positive correlation. It can however be noted that for very low values of $T_{P}$ the relation reverses and higher cost were incurred. This is caused by the EMS ignoring even very high hourly energy import prices if $\rho_{P}$ is large enough in comparison $\rho_{c}$. Economically unfortunate schedules are derived, even if they only lead to a minimal reduction in $T_{P}$.

TABLE 1 ADDITIONAL EVALUATION IN REFERNCE TO PIT SCALING

\begin{tabular}{|c|c|c|c|c|}
\hline & $\rho_{P I T}=1$ & $\rho_{P I T}=2$ & $\rho_{P I T}=6$ & $\rho_{P I T}=24$ \\
\hline $\bar{T}_{P I T}[\mathrm{~h}]$ & 6.74 & 7.84 & 9.27 & 9.72 \\
\hline$\overline{S O C}_{B E S S, 6}$ & 0.77 & 0.80 & 0.85 & 0.86 \\
\hline $\bar{p}_{1}^{\text {Max }}[\mathrm{kW}]$ & 7.28 & 7.33 & 7.32 & 6.37 \\
\hline
\end{tabular}

CONCLUSION

This paper presented the simulation of MG operation in a large-scale parameter variation for a multi-objective MPC based EMS. Specifically, PIT maximization was included in the objectives. Results extend on previous works and show how different EMS objectives always provide a tradeoff in optimality. A reversal of relations between of objectives, that otherwise follow a clear trend, was shown that for large differences in their weight, as in the case of cumulative cost and energy exchange with the main grid.

Additional results show that energy losses in the network can in fact be negatively correlated with both cost and net energy exchange, although counterintuitive, depending on the MG topology. Further, the integration of the explicit PIT maximization as a purely discrete parameter function was shown to negatively implicate several other objectives in the cumulative results, if disconnection from the main grid was determined impossible for singular time steps by the EMS.

\section{ACKNOWLEDGMENT}

The research activity presented in this paper is supported by the European Union through the H2020-LCE2016-SGS-731289 project "InterFlex" (http://interflexh2020.com).

\section{REFERENCES}

[1] R.H. Lasseter, "Microgrids and distributed generation", Journal of Energy Engineering, vol. 133, no. 3, pp. 144-149, 2007 -

[2] L. I. Minchala-Avila, L. E. Garza-Castañón, A. Vargas-Martínez, and Y. Zhang, "A Review of Optimal Control Techniques Applied to the Energy Management and Control of Microgrids", Procedia Computer Science, vol. 52, pp. 780-787, 2015.

[3] O. Palizban, K. Kauhaniemi, and J. M. Guerrero, "Microgrids in active network management - Part I: Hierarchical control, energy storage, virtual power plants, and market participation," Renewable and Sustainable Energy Reviews, vol. 36, no. Supplement C, pp. 428-439, 2014.

[4] M. Diekerhof, F. Peterssen and A. Monti, "Hierarchical Distributed Robust Optimization of Demand Response Services," IEEE Trans. on Smart Grid, in press.

[5] L. Meng, E. R. Sanseverino, A. Luna, T. Dragicevic, J. C. Vasquez, and J. M. Guerrero, "Microgrid supervisory controllers and energy management systems: A literature review," Renewable and Sustainable Energy Reviews, vol. 60, pp. 1263 - 1273, 2016.

[6] A. L. Jaimes, S. Z. Martínez, and C.A. Coello, "An introduction to multiobjective optimization techniques," in Optimization in Polymer Processing, pp. 29-57, 2009.

[7] M. R. Sandgani, and S. Sirouspour, "Priority-Based Microgrid Energy Management in a Network Environment," IEEE Trans. on Sustainable Energy, vol. 9, no. 2, pp. 980 990, 2018.

[8] G. Carpinelli, F. Mottola, D. Proto, and A. Russo, "A MultiObjective Approach for Microgrid Scheduling," IEEE Trans. on Smart Grid, vol. 8, no. 5, pp. 2109 2118, 2017.

[9] D. Mildt, R. Kubo, M. Cupelli, and A. Moni, "Islanding Time Evaluation in Residential Microgrids," Proc. 2018 IEEE PES/IAS PowerAfrica Conference, Cape Town, 2018.

[10] D. Mildt, M. Cupelli, and R. Kubo, "Model Predictive Control for Microgrid Power Management under Forecast Uncertainties," Proc. 2017 IEEJ Joint Technical Meeting on Systems and Smart, ST-1751/SMF-17-50, Tokyo, 2017.

[11] M. Koller, T. Borsche, A. Ulbig, and G. Andersson, "Defining a Degradation Cost Function for Optimal Control of a Battery Energy Storage System," Proc. 2013 IEEE Grenoble Conference, Grenoble, 2013.

[12] S. H. Low, "Convex Relaxation of Optimal Power Flow - Part I: Formulations and Equivalence," IEEE Trans. on Control of Network Systems, vol. 1, no.1, pp. 29-57, 2009.

[13] J. Löfberg, "YALMIP: A toolbox for modeling and optimization in MATLAB," Proc. CACSD Conference, Taipei, 2004

[14] The MathWorks, Inc., "MATLAB and Simulink Student Suite 2017b," Natick, Massachusetts, USA, 2017

[15] Gurobi Optimization LLC, "Gurobi Optimizer Reference Manual," 2018 [Online]. Available: http://www.gurobi.com

[16] S. Papathanassiou, N. D. Hatziargyriou and K. Strunz, "A Benchmark Low Voltage Microgrid Network," Proc. CIGRE Symposium on Power Systems with dispersed generation: technologies, impacts on development, operation and performance, Athens, 2005.

[17] I. Richardson, M. Thomson, D. Infield, and C. Clifford. "Domestic electricity use: A high-resolution energy demand model," Energy and buildings, vol. 42, no. 10, pp. 1878-1887, 2010.

[18] EEX, "Phelix," [Online]. Available: https://www.eex.com/en 